\title{
Engineering molecularly-active nanoplasmonic surfaces for DNA detection via colorimetry and Raman scattering
}

\author{
Esmaeil Heydari ${ }^{\mathrm{a}}$, Samuel Mabbott ${ }^{\mathrm{b}}$, David Thompson ${ }^{\mathrm{b}}$, Duncan Graham ${ }^{\mathrm{b}}$, Jonathan M. Cooper ${ }^{\mathrm{a}}$, \\ Alasdair W. Clark*a \\ ${ }^{a}$ Biomedical Engineering Research Division, School of Engineering, University of Glasgow, UK \\ G128LT; ${ }^{b}$ Department of Chemistry, University of Strathclyde, UK \\ *Alasdair.clark@glasgow.ac.uk; phone +44 141330 3216; \\ www.gla.ac.uk/schools/engineering/staff/alasdairclark/
}

\begin{abstract}
We report a novel nanophotonic biosensor surface capable of both colorimetric detection and Raman-scattered detection of DNA infection markers at extreme sensitivities. Combining direct-write lithography, dip-pen nanolithography based DNA patterning, and molecular self-assembly, we create molecularly-active plasmonic nanostructures onto which metallic nanoparticles are located via DNA-hybridization. Arraying these structures enables optical surfaces that change state when contacted by specific DNA sequences; shifting the surface color while simultaneously generating strong Raman-scattering signals. Patterning the DNA markers onto the plasmonic surface as micro-scale symbols results in easily identifiable color shifts, making this technique applicable to multiplexed lab-on-a-chip and point-of-care diagnostic applications.
\end{abstract}

Keywords: Plasmonics, Biosensors, DNA assembly, Colorimetry, Naked-eye detection, Surface Enhanced Raman Spectroscopy, Electron-beam Lithography, Dip-pen Nanolithography

\section{INTRODUCTION}

Excitation of localized surface plasmon resonances (LSPR) in metallic nanoparticles has allowed researchers to control light beyond the diffraction limit, and has driven the development of new nano-scale technologies in the biomedical sciences. ${ }^{1,2}$ Acting as nano-antenna for light radiation, plasmonic particles can be used as a matching interface between far-field and near-field optics. ${ }^{3,4}$ Tuned by altering their size and shape, these particles can be engineered to exhibit extraordinary scattering, absorption, and focusing properties, making them promising for applications requiring selective light emission and/or harvesting. ${ }^{5-8}$ One such application is the use of engineered plasmonic nanostructures for selective color filtering; tailoring their optical properties to control the wavelengths of visible light they scatter, transmit or absorb. ${ }^{9-}$ 11

In addition to geometric tuning to define plasmonic color, it is also possible to control the optical properties of plamonic nano-metals by bringing discrete structures in close contact with one another $(<2 \mathrm{~nm}$ such that their free-electron clouds can interact). ${ }^{12-15}$ Plasmonic coupling of this kind has led to series of exciting applications that would not be possible using single nanoparticles alone. ${ }^{16}$ One route towards engineering these coupled plasmonic systems is the use of molecular affinity binding to control the assembly and spacing of complex nanoparticle systems. ${ }^{17-19}$ However, to date these schemes have largely been confined to the solution phase, with little effort directed toward the combination of engineered plasmonic surfaces and molecularly-active plasmonic particles. ${ }^{10,11,20,21}$

In this paper, we combine electron-beam lithography, dip-pen nanolithography and DNA-mediated nanoparticle assembly to create a plasmonic metasurface that changes color when exposed to DNA markers for a particular fungal infection. The arbitrary patterning control afforded by DPN allows these modifications to take the form of micro-scale images, lettering or symbols. Completion of a DNA sandwich assay results in nanoparticle localization onto these patterned areas if the specific infection marker for that pattern was present. The resultant interaction between the plasmonic surface and the metallic nanoparticles shifts the plasmonic resonance conditions in these areas, revealing the pattern as a change in surface color. Adding a Raman-active dye to the nanoparticle label allows further detection versatility through Raman mapping of the surface. We demonstrate that these molecularly-active plasmonic surfaces hold significant potential in lab-on-a-chip and point-of-care diagnostic tools; delivering dynamic optical surfaces with visual, colorimetric output that can identify DNA at extreme sensitivities, and are observable without the need for specialized equipment, or even a power supply. 


\section{METHODS}
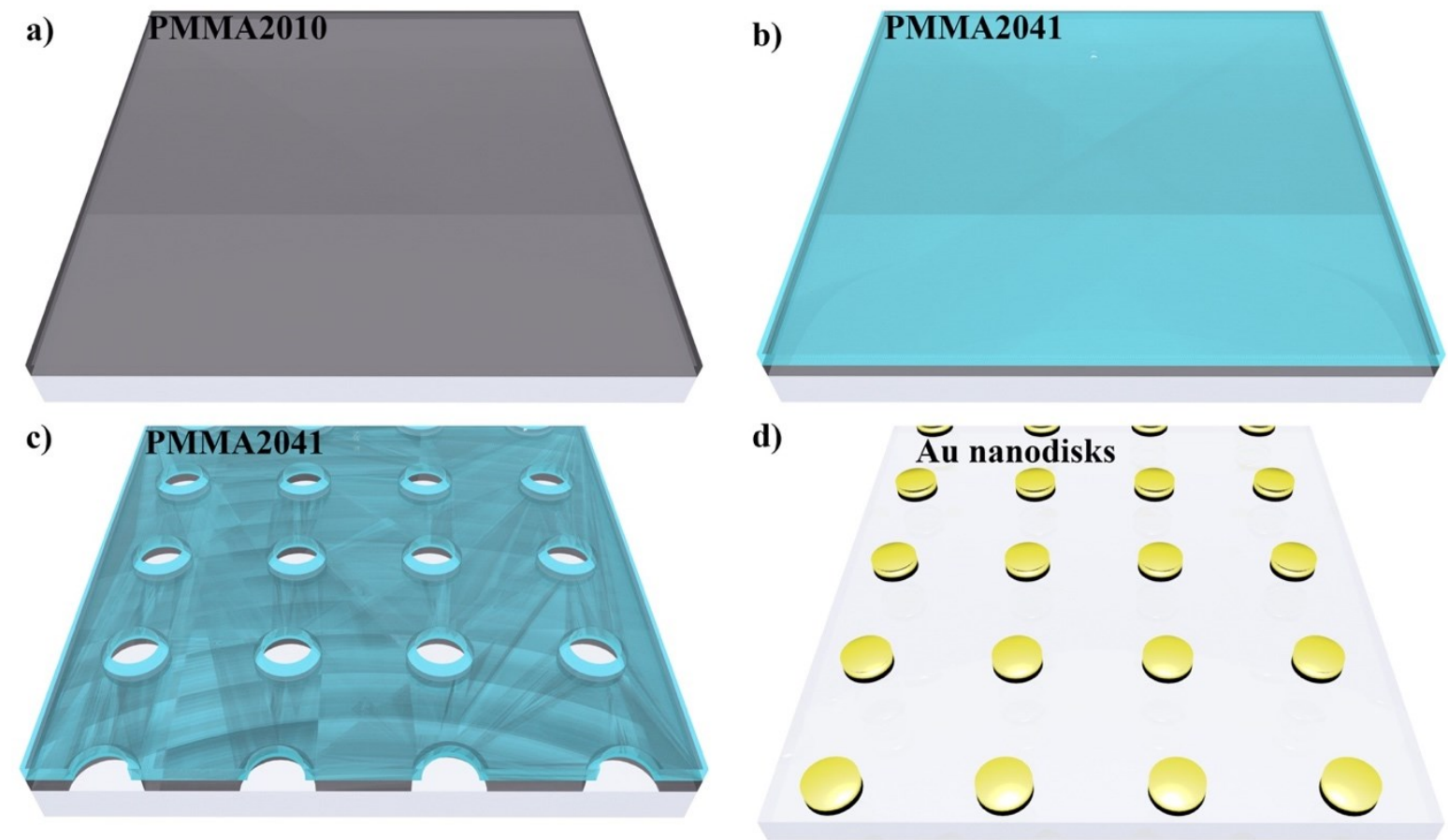

Figure 1. Fabrication, via electron-beam lithography, of Au nanodisks which comprise the plasmonic metasurface . a) and b) Deposition of an 80nm PMMA bi-layer. c) Electron-beam exposure and development of the PMMA. d) Deposition of $2 / 25 \mathrm{~nm}$ of Ti/Au layer and lift-off of the excess resist.

Standard electron-beam lithography processes were used to fabricate Au nanodisks on the surface of a glass substrate. A bi-layer of Poly(methyl methacrylate) (PMMA) resist was spun onto a $500 \mu \mathrm{m}$ Pyrex glass substrate (Figure 1a and b) and subsequently patterned with a $500 \mu \mathrm{m} \times 500 \mu \mathrm{m}$ array of $120 \mathrm{~nm}$ diameter disks (period 250nm) using a Vistec VB6 UHR EWF electron beam lithography tool. A $2 / 25 \mathrm{~nm}$ bi-layer of Ti/Au was deposited onto the surface via electron beam metal evaporation before lift-off was performed in warm acetone (Figure 1d). 


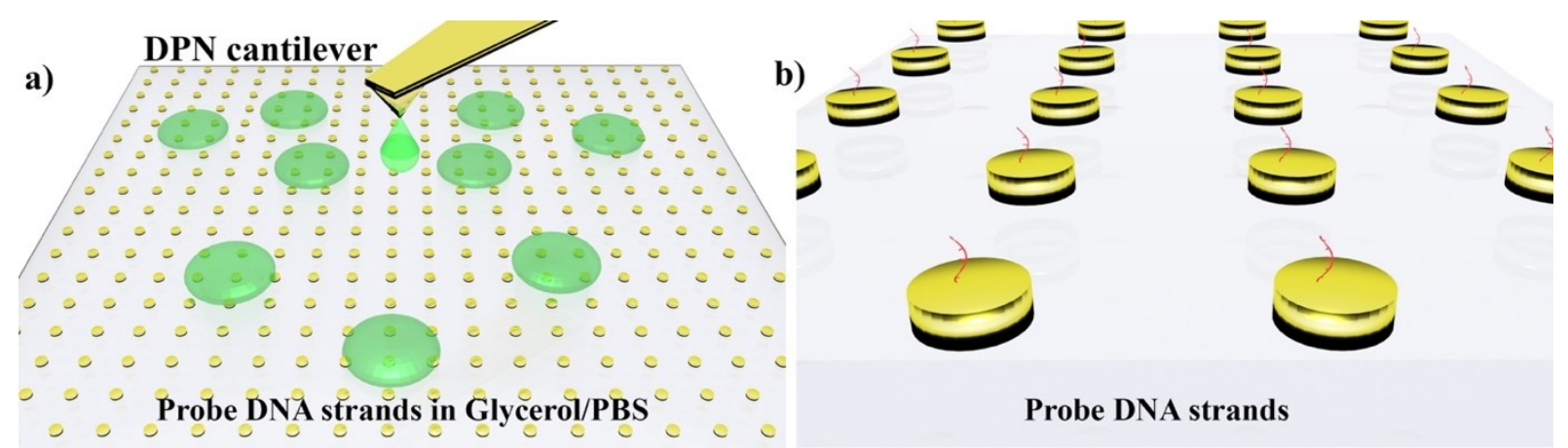

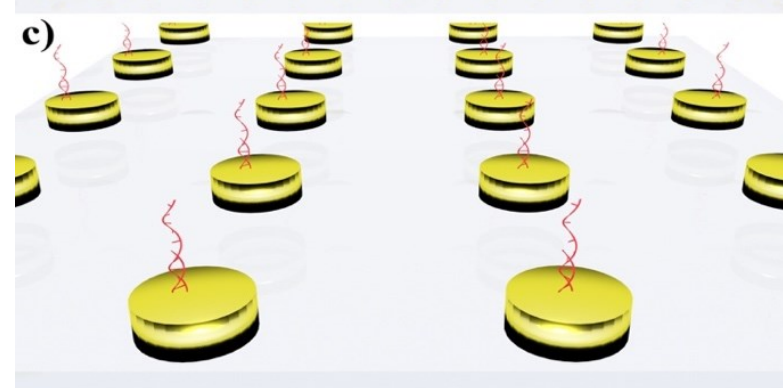

Target and probe DNA strands hybridisation

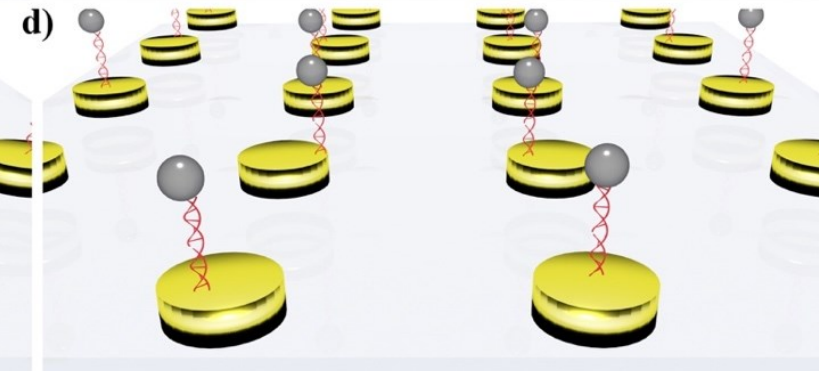

Label, target and probe DNA strands hybridisation

Figure 2. DNA patterning and detection a) DNA patterns defined on the surface using Dip-pen nanolithography. 10\%(v/v) solutions of Glycerol/ probe DNA stands were used. b) The thiolated DNA probe sequences attached to the Au nanodisks. c) Target DNA relating to the fungal infection is captured by the probe strands. d) The nanoparticle-tagged DNA is added resulting in $\mathrm{Ag}$ nanoparticle localization where the target DNA is present.

The plasmonic array was then specifically patterned with DNA probe sequences relating to the fungal infection Aspergillus Fumigatus using dip-pen nanolithography, Figure 2. The probe strand had the sequence, 5'-ATC ACC TCT CGG-HEGHEG-HEG-SH-3', and was modified with a reduced thiol group on the $3^{\prime}$ end to allow attachment to the Au disks on the substrate surface (all DNA was sourced from ATD Bio Ltd.). Using the DPN to modify the surface allows us to specifically control which areas of the colored plasmonic pattern are molecularly active (ie. which areas will respond to the presence of the target DNA which indicates the presence of the infection). After washing the target DNA was then introduced to the surface, (sequence 5'-CCG AGA GGT GAT ACA TTC CGA GGG-3'). The sample was washed again before, finally, a nanoparticle labelled strand complimentary to the $2^{\text {nd }}$ half of the target strand was introduced (sequence, 5'-SH-HEG-HEGHEG-CCC TCG GAA TGT-3', attached to a $40 \mathrm{~nm}$ Ag nanoparticle via a thiol group on the 5' end (a nanoparticle which was also modified with the Raman active dye malachite green). As a result, on completion of the assay the gold nanodisks on the surface that were initially modified using DPN were now decorated with silver nanoparticles. Optical characterization of the plasmonic surface was performed using absorption spectroscopy (Shimadzu UV3101PC), brightfield microscopy (Zeiss Axio Imager A1 in combination with a 10x 0.25NA objective lens), and Raman spectroscopy (Horiba Jobin Yvon LabRam INV spectrometer with a 633nm HeNe laser). 


\section{RESULTS AND DISCUSSION}
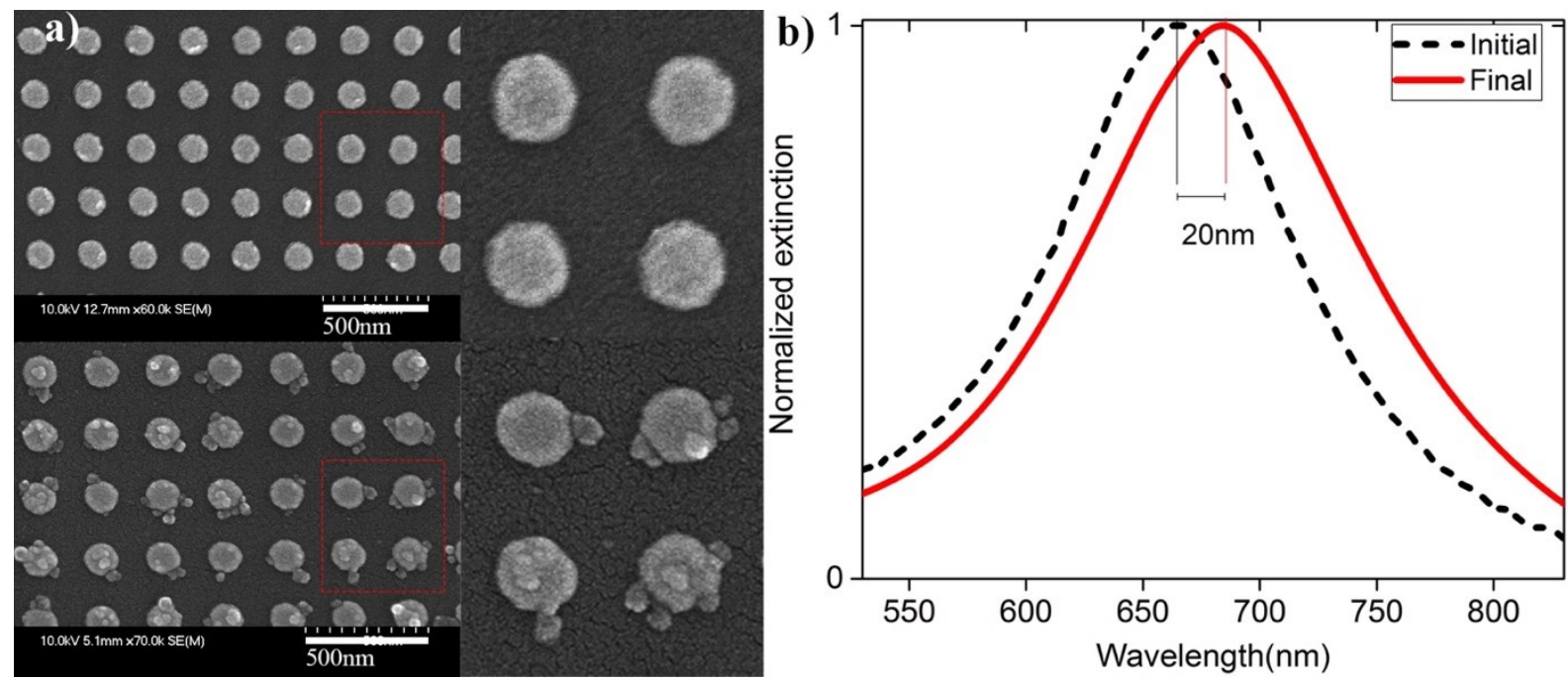

Figure 3. SEM images and plasmon resonance band of the biosensor before and after DNA detection. a) SEM image of the nanodisks array before DNA detection (top), and after DNA detection (top) showing the Ag nanoparticles coupled to the Au nanodisks. b) Plasmon resonance band of the nanophotonic biosensor before and after detection.

Figure 3a shows SEM images of the surface of the plasmonic biosensor before and after detection of the target DNA. After running the assay, the disks modified with the DNA probes have been decorated with Ag nanoparticles. The result of this decoration is plasmonic coupling between the Ag and Au structures; coupling which leads to a red-shift of the LSPR band for those structures (Figure $3 \mathrm{~b}$ ). The extent of the wavelength shift depends on the number of Ag-NPs bound to the Au nanodisks. ${ }^{21,22}$ The $20 \mathrm{~nm}$ shift observed here related to an average of $3 \mathrm{Ag}$ nanoparticles bound to each Au nanodisk.

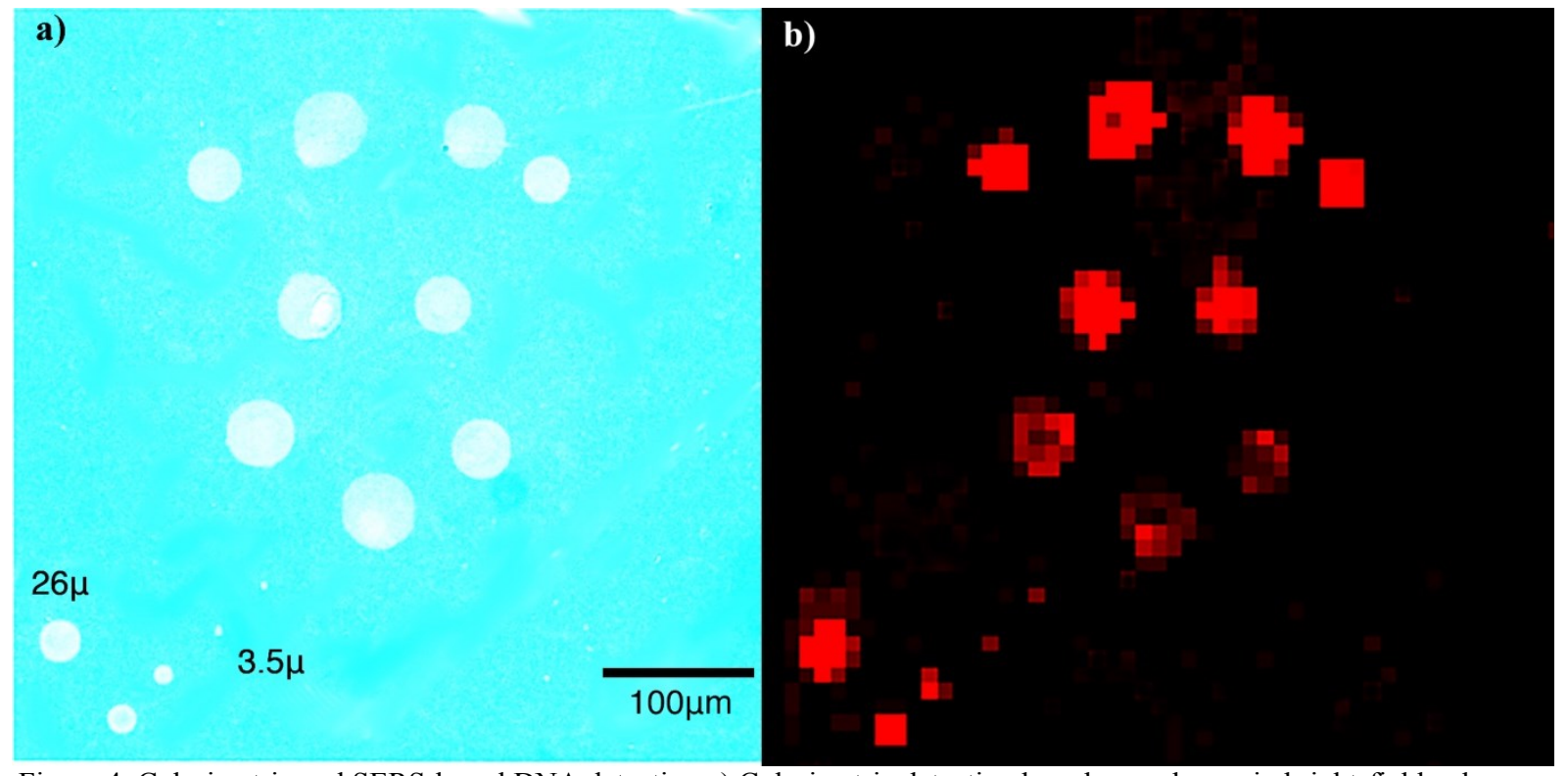

Figure 4. Colorimetric and SERS-based DNA detection a) Colorimetric detection based on a change in bright-field color (blue to red) of the nanophotonic biosensor surface where the initial DPN-defined DNA pattern was established. In this case, a simple arrangement of DNA spots has been patterned in the shape of a smiling face (middle) and a check-mark (bottom left) b) The same pattern interrogated with Raman mapping (using the $1617 \mathrm{~cm}^{-1}$ band of malachite green).

Figure 4a shows a bright-field image of the biosensor after detection. The smiley face, in the middle, and check mark, in the bottom left corner, are a result of plasmonic coupling between the Au nanodisks and the Ag nanoparticles. The plasmonic surface was specifically engineered to appear blue if no Ag particles were present, changing to a red color upon 
DNA-mediated particle binding. A secondary detection technique used to further confirm the presence of the target DNA was SERS (Figure $4 \mathrm{~b}$ ). In this case the electric-field enhancement generated at the interface between the Au disk and Ag particle as a consequence of plasmonic coupling was used to measure the presence of malachite-green on the $\mathrm{Ag}$ particle. As can be seen from Figure 4, both the colorimetric measurement and the SERS map agree with one another, confirming that the color change seen in Figure $4 \mathrm{a}$ is due to the presence of target DNA.

This novel implementation of a colorimetric sensor has, in its combination of plasmonic metasurfaces and DPN molecular patterning, the ability to specifically pattern different areas of the sensor with different DNA probes. As a result, this system holds the potential to be extended beyond the single infection sensor we have demonstrated here, to a multiplexed system where different patterns/symbols are assigned to different DNA sequences of interest. As a colorimetric sensor operating in bright-field conditions and without the need for a power supply, specialized optics, or operators with technical or medical training (the sensor can be engineered to literally spell out the diagnosis), this technology, and its multiplexing potential, holds significant promise for future incorporation into point-of-care diagnostic systems.

\section{CONCLUSION}

We have demonstrated a novel plasmonic biosensor metasurface for simple colorimetric detection of DNA targets. Using DPN to build molecularly active areas on the plasmonic surface allows us to define arbitrary patterns which change color in the presence of particular disease and infection markers. These areas can take the form of words or symbols in order to maximize interpretability by the user, making this technology applicable to multiplexed point-of-care diagnostics.

\section{ACKNOWLEDGMENTS}

The work was supported by the Royal Academy of Engineering (grant number 10216/103). The authors wish to thank all the staff working in the James Watt Nanofabrication Centre for their support.

\section{REFERENCES}

[1] Anker, J. N., Hall, W. P., Lyandres, O., Shah, N. C., Zhao, J. and Van Duyne, R. P., "Biosensing with plasmonic nanosensors," Nat. Mater. 7, 442-453 (2008).

[2] Bozhevolnyi, D. K. and Gramotnev, S. I., "Plasmonics beyond the diffraction limit," Nat. Photonics 4, 83-91 (2010).

[3] Mühlschlegel, P., Eisler, H. J., Martin, O. J. F., Hecht, B. and Pohl, D. W., "Resonant optical antennas," Science 308(5728), 1607-1609 (2005).

[4] Bharadwaj, P., Deutsch, B. and Novotny, L., "Optical antennas," Adv. Opt. Photon. 1(3), 438-483 (2009).

[5] Atwater, H. A. and Polman, A., "Plasmonics for improved photovoltaic devices," Nat. Mater. 9, $205-213$ (2010).

[6] Knight, M.W., Sobhani, H., Nordlander, P. and Halas, N. J., "Photodetection with active optical antennas," Science 332(6030), 702-704 (2011).

[7] Heydari, E., Flehr, R. and Stumpe, J., "Influence of spacer layer on enhancement of nanoplasmon-assisted random lasing," Appl. Phys. Lett. 102(13), 133110 (2013).

[8] Heydari, E., Pastoriza-Santos, I., Flehr, R., Liz-Marzán, L. M. and Stumpe, J., "Nanoplasmonic enhancement of the emission of semiconductor polymer composites," J. Phys. Chem. C 117(32), 16577-16583 (2013).

[9] Li, Z., Clark, A. W. and Cooper, J. M., "Dual color plasmonic pixels create a polarization controlled nano color palette," ACS Nano, 10.1021/acsnano.5b05411 (2016).

[10] Clark, A. W. and Cooper, J. M., "Plasmon shaping by using protein nanoarrays and molecular lithography to engineer structural color," Angew. Chem. 124, 3622-3626 (2012).

[11] Clark, A. W., Thompson, D. G., Graham, D. and Cooper, J. M., "Engineering DNA binding sites to assemble and tune plasmonic nanostructures," Adv. Mater. 26, 4286-4292 (2014).

[12] Sönnichsen, C., Reinhard, B. M., Liphardt, J. and Alivisatos, A. P., "A molecular ruler based on plasmon coupling of single gold and silver nanoparticles," Nat. Biotechnol. 23, 741-745 (2005).

[13] Ghosh, S. K. and Pal, T., "Interparticle coupling effect on the surface plasmon resonance of gold nanoparticles: from theory to applications," Chem. Rev. 107(11), 4797-4862 (2007).

[14] Aizpurua J., Bryant, G. W., Richter, L. J., García de Abajo, F. J., Kelley, B. K. and Mallouk, T., "Optical properties of coupled metallic nanorods for field-enhanced spectroscopy," Phys. Rev. B 71, 235420 (2005). 
[15] Gunnarsson, L., Rindzevicius, T., Prikulis, J., Kasemo, B., Käll, M., Zou, S. and Schatz, G. C., "Confined plasmons in nanofabricated single silver particle pairs: experimental observations of strong interparticle interactions," J. Phys. Chem. B 109(3), 1079-1087 (2005).

[16] Mirkin, C. A., Letsinger, R., Mucic, R. C. and Storhoff, J. J., "A DNA-based method for rationally assembling nanoparticles into macroscopic materials," Nature 382, 607-609 (1996).

[17] Fan, J. A., Wu, C., Bao, K., Bao, J., Bardhan R., Halas, N. J., Manoharan V. N., Nordlander, P., Shvets, G. and Capasso, F., "Self-assembled plasmonic nanoparticle clusters," Science 328 (5982), 1135-1138 (2010).

[18] Tan, S. J., Campolongo, M. J., Luoa, D. and Chenga, W., "Building plasmonic nanostructures with DNA," Nat. Nanotechnol. 6, 268-276 (2011).

[19] Kuzyk, A., Schreiber, R., Fan, Z., Pardatscher, G., Roller, E.-M., Högele, A., Simmel, F. C., Govorov, A. O. and Lied, T., "DNA-based self-assembly of chiral plasmonic nanostructures with tailored optical response," Nature 483, 311-314 (2012).

[20] Heydari, E., Thompson, D., Graham, D., Cooper, J. M. and Clark, A. W., "An engineered nano-plasmonic biosensing surface for colorimetric and SERS detection of DNA-hybridization events," Proc. of SPIE 9340, 93400O1-93400O6 (2015).

[21] Clark, A. W. and Cooper, J. M., "Molecularly defined plasmonic engineering to visualize antibody binding events by eye, " Proc. of SPIE 8597, 85970Q1-85970Q7 (2013).

[22] Waldeisen, J. R., Wang, T., Ross, B. M. and Lee, L. P., "Disassembly of a core-satellite nanoassembled substrate for colorimetric biomolecular detection," ACS Nano 5(7), 5383-5389 (2011).

[23] Ross, B. M., Waldeisen, J. R., Wang, T. and Lee, L. P., "Strategies for nanoplasmonic core-satellite biomolecular sensors: Theory-based Design," Appl. Phys. Lett. 95, 193112 (2009). 